\title{
A. HAURIE
}

A. M. DUSS AIX

\section{Un modèle dynamique de négociation sous forme d'un jeu semi-différentiel}

Revue française d'automatique, d'informatique et de recherche opérationnelle. Recherche opérationnelle, tome 6, n V1 (1972), p. 65-86.

<http://www.numdam.org/item?id=RO_1972_6_1_65_0>

(C) AFCET, 1972, tous droits réservés.

L'accès aux archives de la revue « Revue française d'automatique, d'informatique et de recherche opérationnelle. Recherche opérationnelle » implique l'accord avec les conditions générales d'utilisation (http://www.numdam.org/ legal.php). Toute utilisation commerciale ou impression systématique est constitutive d'une infraction pénale. Toute copie ou impression de ce fichier doit contenir la présente mention de copyright.

\section{Numdam}

Article numérisé dans le cadre du programme

Numérisation de documents anciens mathématiques

http://www.numdam.org/ 


\title{
UN MODELE DYNAMIQUE DE NEGOGIATION SOUS FORME D'UN JEU SEMI-DIFFERENTIEL(1)
}

\author{
par A. Haurie ( ${ }^{2}$ ) et A. M. Dussaix ( $\left.{ }^{3}\right)$
}

\begin{abstract}
Sommaire. - Les négociations syndicales ont une structure de jeux dynamique. La théorie des jeux différentiels développée par Isaacs [12], Blaquière et Leitmann [3] 'et Case [6] n'est pas directement utilisable pour résoudre de tels jeux (cf. Case [7]).

Dans cette étude, nous proposons un modèle de négociation à deux joueurs $E$ et $S$ où $S$ peut faire évoluer ses exigences dans le temps cependant que E décide seul de l'instant d'acceptation des revendications de $S$. Le gain des deux joueurs depend alors du point d'accord et de l'instant où cet accord est intervenu. Nous montrons que la notion d'équilibre de Nash est un critère d'optimalité réaliste, que la notion de point efficace par contre est irréaliste. Nous donnerons ensuite une définition précise du type de jeu illustré par cette négociation et nous établirons rigoureusement certains résultats obtenus en utilisant la terminologie des jeux différentiels.
\end{abstract}

\section{$1^{\text {re }}$ PARTIE : UN MODELE DYNAMIQUE DE NEGOCIATION}

\section{Introduction}

Les théories classiques de la négociation ne tiennent pas compte de l'influence du temps. Elles indiquent, s'il y a accord, ce que sera cet accord (Nash, Shapley) et les propriétés qu'il doit avoir. Ces théories ne considèrent pas la durée de la négociation ni l'influence de cette durée sur le résultat final.

Quelques auteurs se sont intéressés récemment à ce problème. On a alors construit des modèles où les demandes des joueurs évoluent dans le temps et où il $\mathrm{y}$ a accord quand ces demandes sont compatibles (Hicks-Case [7]).

La variation des demandes d'un joueur peut être considérée comme autonome, c'est-à-dire suivre une certaine courbe d'apprentissage (cf. Ashenfelter et Johnson [1]), cependant, il est certain qu'il y a interdépendance entre les concessions de chacun des joueurs et que la demande de chaque joueur va

(1) Cette étude a bénéficié du soutien financier du Conseil des Arts du Canada (Fonds $n^{\circ}$ S70 1491) et de l'Ecole des Hautes Etudes Commerciales de Montréal.

(2) Professeur à l'Ecole des Hautes Etudes Commerciales de Montréal.

(3) Actuellement à I'E.S.S.E.C., Mme Dussaix était professeur à l'Ecole des Hautes Etudes Commerciales de Montréal lors de la réalisation de cette étude.

Revue Française d'automatique, Informatique et Recherche Opérationnelle n० V-1, 1972. 
dépendre de la façon dont il perçoit le comportement, c'est-à-dire le degré de résistance, de son adversaire (cf. J. G. Cross [10]). Dans un modèle dynamique de négociation, le problème important est l'évaluation par chaque joueur du taux de concession de l'adversaire.

Le modèle que nous présentons décrit une négociation entre un syndicat et la direction d'une entreprise lors d'une grève. L'entreprise $E$ ne formule pas d'offres de salaire mais refuse ou accepte les demandes du syndicat $S$. Dans ce dernier cas, la grève cesse. Nous nous trouvons dans un cas où seulement un joueur peut concéder de façon "continue » par une réduction de ses revendications, tandis que l'autre joueur, lorsqu'il concède, met un terme au jeu.

\section{Un modèle élémentaire à gain terminal pour chaque joueur}

Nous considérons une entreprise $E$ dont tous les employés sont groupés dans un syndicat $S$. Le nombre d'employés et l'équipement de l'entreprise étant fixés, cette entreprise a une production potentielle, engendrant un revenu potentiel net des coûts de production autres que celui de la main-d'œuvre, de $\$ y$ par jour.

Le syndicat $S$ va revendiquer une part $d$ de ce revenu potentiel quotidien, comme masse de salaires versés aux employés. L'entreprise $E$ peut accepter ou refuser cette revendication. Il existe une période de temps fixée $0 \leqslant t \leqslant T$, où $T$ est l'horizon commun aux deux parties.

$\mathrm{Si}$, à l'instant $t^{f}$, la revendication syndicale $d^{f}$ est acceptée par $E$ alors les deux parties auront pour gains respectifs :

$$
\begin{aligned}
& g_{S}=d^{f}\left(T-t^{f}\right) \\
& g_{E}=\left(y-d^{f}\right)\left(T-t^{f}\right) .
\end{aligned}
$$

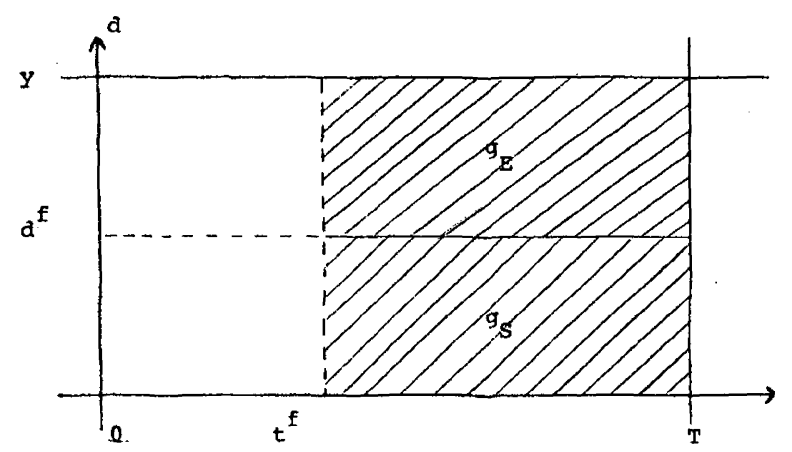

Figure 1

Nous voyons alors que ce gain dépend explicitement de $t^{f}$, instant de l'accord. 
En l'absence de tout autre composante dans les gains des deux joueurs, le choix d'un point $\left(t^{f}, d^{f}\right)$ représentant un arbitrage « optimal » est un problème comportant toutes les difficultés d'un problème classique de marchandage.

Il est facile de constater que, en l'absence de contraintes, chaque partie, pour une revendication $d^{f}$ fixée a intérêt à ce que l'accord intervienne au plus tôt. En particulier, l'ensemble des points $\left(t^{f}, d^{f}\right)$ «non dominés ", c'est-à-dire tels qu'il soit impossible de trouver un autre accord améliorant le gain de chaque joueur est défini par :

$$
\mathcal{C} \triangleq\left\{\left(0, d^{\circ}\right) \quad: \quad 0 \leqslant d^{\circ} \leqslant y\right\}
$$

De façon normative, on a l'habitude de requérir l'optimalité parétienne pour les solutions d'un problème de marchandage; dans ce jeu dynamique cela parait impossible.

En effet, toute négociation prend du temps. Ceci se traduit par un coût et, si l'entreprise refuse la demande $d^{\circ}$ du syndicat au début de la négociation, un accord ne sera plus pareto-optimal. Même si on renonce à cette condition, on aura beaucoup de difficulté à découvrir les points $\left(t^{f}, d^{f}\right)$ acceptables par les deux parties.

Nous pourrions considérer que chaque joueur procède à des évaluations subjectives de ses degrés de conviction quant à l'acceptabilité d'une revendication $d$ avant une date $\tau \leqslant T$. Le problème fondamental est évidemment celui de définir des procédures d'évaluation de ces degrés de conviction (cf. [9]).

Dans les paragraphes suivants, nous montrerons que l'adjonction de contraintes temporelles sur le comportement de $S$ ainsi que la prise en compte de l'histoire de la négociation dans la fonction d'utilité de $S$ permettent de bâtir un modèle beaucoup plus intéressant, faisant apparaître des solutions bien définies au problème de négociation.

\section{Modèle dynamique de négociation sous forme d'un jeu semi-différentiel}

3.1 Contraintes dynamiques et influence de l'histoire de la négociation sur l'utilité de $S$

L'histoire d'une négociation sera représentée par une fonction

$$
\left(d(t) ; 0 \leqslant t \leqslant t^{f}\right)
$$

décrivant l'évolution dans le temps des revendications syndicales.

Nous supposerons que les syndiqués déterminent deux revendications :

$d^{\circ}$ revendication initiale

$d^{*}$ revendication minimale au-dessous de laquelle les dirigeants syndicaux ne peuvent descendre en aucun cas. 
Les dirigeants syndicaux, chargés de la négociation avec $E$, peuvent réduire la revendication des syndiqués suivant un processus d'apprentissage représenté par l'équation différentielle suivante :

$$
\dot{d}(t)=-u\left(d(t)-d^{*}\right) .
$$

Selon cette relation la variation instantanée de la revendication des syndiqués est proportionnelle à l'écart existant entre la revendication actuelle $d(t)$ et la revendication minimale $d^{*}$. Le coefficient de proportionnalité, - $-u$, est une variable permettant aux dirigeants syndicaux de contrôler l'apprentissage des syndiqués.

La courbe d'apprentissage n'est donc pas une donnée institutionnelle mais représente un comportement « intelligent » du syndicat.

Nous supposerons que la variable de contrôle $u$ est soumise aux contraintes suivantes :

$$
0 \leqslant u \leqslant \tau
$$

où $\tau$ est le coefficient d'apprentissage maximal des syndiqués.

Les relations (1) et (2) constituent des contraintes dynamiques sur le comportement de $S$.

L'histoire d'une négociation sera alors définie par la donnée de la revendication initiale $d^{\circ}$, d'une politique de contrôle de l'apprentissage des syndiqués, décrite par la fonction :

$$
\left(u(t) ; 0 \leqslant t \leqslant t^{f}\right),
$$

engendrant la fonction $\left(d(t) ; 0 \leqslant t \leqslant t^{f}\right)$ solution de (1) avec la condition initiale $d(0)=d^{\circ}$. L'instant $t^{f}$, date d'intervention d'un accord, est choisi par l'entreprise $E$.

L'histoire de la négociation peut être représentée graphiquement ainsi :

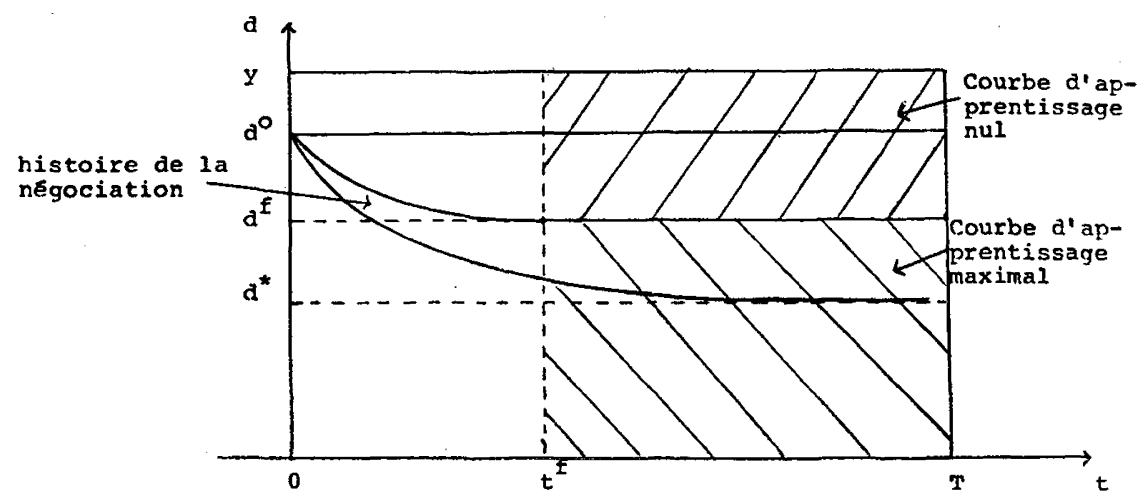

Figure 2

Revue Française d'Automatique, Informatique et Recherche Opérationnelle 
Le graphe de la fonction $\left(d(t) ; 0 \leqslant t \leqslant t^{f}\right)$ est compris entre deux courbes d'apprentissage : celle d'apprentissage nul, correspondant à $u \equiv 0$, et celle d'apprentissage maximal correspondant à $u \equiv \tau$. La première courbe est représentée par la droite.

$$
\underset{n u l}{d} \equiv d^{\circ}
$$

la seconde est représentée par l'exponentielle :

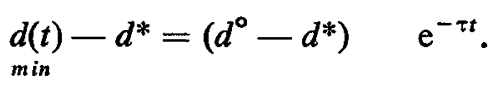

Nous appellerons $K_{d_{o}}$ l'ensemble fermé délimité par ces deux courbes : c'est l'ensemble des points d'accord possibles, étant donnée une demande initiale $d^{\circ}$.

Il est important de constater que, dans ce processus de négociation, les décisions du syndicat sont représentées par $d^{\circ}$, puis par la fonction $u(t)$, cependant que la décision de $E$ est représentée par le choix de $t^{f}$ et donc par l'acceptation de la revendication $d^{f}=d\left(t^{f}\right)$ à l'instant $t_{f}$.

Nous pourrons préciser l'histoire de la négociation si cette histoire a une influence sur l'utilité de $S$.

Nous supposerons que le fait de s'éloigner de la revendication initiale comporte une désutilité pour $S$ (preuve de faiblesse, etc...). Cela peut être traduit par la considération du gain suivant pour $S$ :

$$
g_{S}=d\left(t^{f}\right)\left(T-t^{f}\right)+\alpha \int_{0}^{t^{f}}\left(d(t)-d^{\circ}\right) d t \quad ; \quad \alpha>0
$$

ou, un peu plus généralement :

$$
g_{S}=d\left(t^{f}\right)\left(T-t^{f}\right)+\alpha \int_{0}^{t^{f}} \varphi_{S}\left(d(t)-d^{\circ}\right) d t \quad ; \quad \alpha>0
$$

où $\varphi_{S}(\cdot)$ est une fonction monotone croissante.

Dans l'optique de J. H. Case [7] on peut considérer que $g_{s}$ traduit les préférences agrégées des deux groupes distincts que sont les syndiqués d'une part et les chefs syndicaux d'autre part. L'objectif des chefs syndicaux est de maximiser le gain $\left(T-t^{f}\right) d^{f}$ résultant de l'accord final (sinon, une fois la négociation terminée, les syndiqués pourraient leur reprocher de ne pas avoir conclu l'accord au bon moment). Les membres du syndicats sont d'autre part hostiles à toute concession. 
3.2 Construction de l'ensemble des points d'accord acceptables pour $E$ Considérons donc le modèle de négociation défini par le jeu suivant :

i) fonctionnelles de gain :

$$
\begin{aligned}
& g_{S}=d\left(t^{f}\right)\left(T-t^{f}\right)+\alpha \int_{0}^{t^{f}}\left(d(t)-d^{\circ}\right) d t \\
& g_{E}=\left(y-d^{f}\right)\left(T-t^{f}\right)
\end{aligned}
$$

ii) contraintes :

$$
\begin{aligned}
& \dot{d}(t)=-u\left(d(t)-d^{*}\right) \\
& 0 \leqslant u \leqslant \tau \\
& d(0)=d^{\circ} \\
& d\left(t^{f}\right)=d^{f} \\
& t^{f} \leqslant T
\end{aligned}
$$

iii) Vạriable de contrôle à la disposition de $S$ :

$$
(u(t) ; t \geqslant 0): \text { coefficient d'apprentissage. }
$$

iv) Variable de contrôle à la disposition de $E$ :

$t^{f}:$ instant de l'accord entre les parties.

Un point $\left(t^{f}, d^{f}\right)$ sera acceptable pour $E$ seulement si le gain marginal de $E$ quand il attend $d t$ jours de plus s'annule. En effet $E$ est seul à choisir l'instant terminal donc, seules des considérations relative au gain de $E$ permettent de déterminer $t^{f}$.

Nous avons, en dérivant l'expression (5) :

$$
\frac{d g_{E}}{d t}=\left(d^{f}-y\right)-\dot{d}\left(t^{f}\right)\left(T-t^{f}\right)
$$

et nous constatons que pour trouver le lieu des points où $\frac{d g_{E}}{d t}$ s'annule, nous devons supposer que $E$ connaît le taux de concession final $\dot{d}\left(t^{f}\right)$. Pour cela, il suffit de supposer que $E$ connait le coefficient d'apprentissage maximal, $\tau$, ainsi que la forme générale de l'influence de l'histoire de la négociation sur l'utilité de $S$. Si $E$ sait que la fonction de gain de $S$ a la forme donnée en (4), il en concluera que nécessairement $\dot{d}\left(t^{f}\right)=-\tau\left(d\left(t^{f}\right)-d^{*}\right)$.

En effet, pour passer de la revendication initiale à la revendication $d^{f}$ au temps $t^{f}, S$ choisira une courbe d'apprentissage admissible (compatible avec 
les contraintes (1) et (2)) rendant minimale la surface $\Sigma(d)$ comprise entre la droite : $d \equiv d^{\circ}$ et le graphe de $d(t)$.

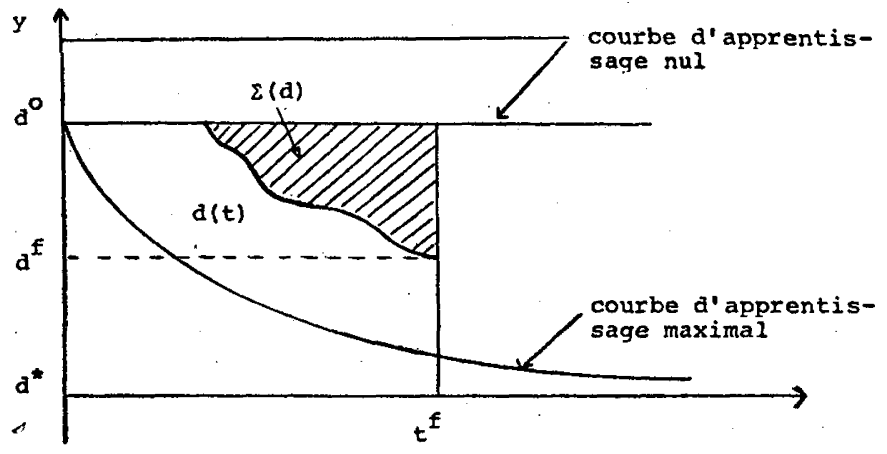

Figure 3

Un calcul variationnel simple nous montre que la fonction $\left(u(t) ; 0 \leqslant t \leqslant t^{f}\right)$ engendrant la fonction $\left(d(t) ; 0 \leqslant t \leqslant t^{f}\right)$ rendant minimale la surface $\Sigma(d)$ est nécessairement de la forme suivante :

$$
\left\{\begin{array}{lll}
u(t)=0 & \text { si } & 0 \leqslant t \leqslant t^{c} \\
u(t)=\tau, & \text { si } & t^{c} \leqslant t \leqslant t^{f}
\end{array}\right.
$$

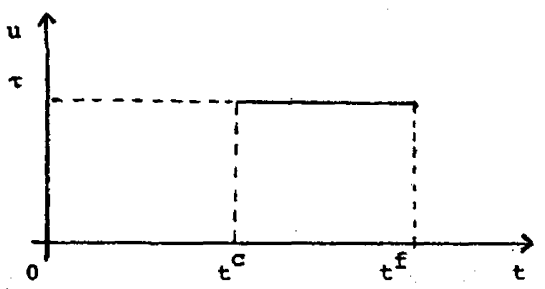

Figure 4

On en conclut donc que $S$ adoptera nécessairement une politique d'intransigeance entre les instants 0 et $t^{c}$ puis une politique de concession maximale àlpartir de l'instant de commutation $t^{c}$.

$E$ connaît donc le taux de variation des exigences de $S$ en tout point $\left(t^{f}, d^{f}\right)$ ou $d^{f}$ est inférieur à $d^{\circ}$ :

$$
\dot{d}\left(t^{f}\right)=-\tau\left(d^{f}-d^{*}\right) .
$$

En portant (8) dans l'expression (6) nous obtenons :

$$
\frac{d g_{E}}{d t}=d^{f}-y+\tau\left(d^{f}-d^{*}\right)\left(T-t^{f}\right.
$$

$n^{\circ} \mathrm{V}-1,1972$. 
et le lieu des points acceptables pour $E$ est alors défini par

$$
\Xi * \triangleq\left\{\left(t^{f}, d^{f}\right): \frac{d g_{E}}{d t}=0\right\}
$$

c'est-à-dire l'hyperbole d'équation :

$$
\theta^{*}\left(t^{f}, d^{f}\right) \triangleq T-t^{f}-\frac{y-d^{f}}{\tau\left(d^{f}-d^{*}\right)}=0
$$

Nous appellerons l'ensemble $\Xi^{*}$ la cible de $S$. Nous l'avons illustrée sur la figure suivante :

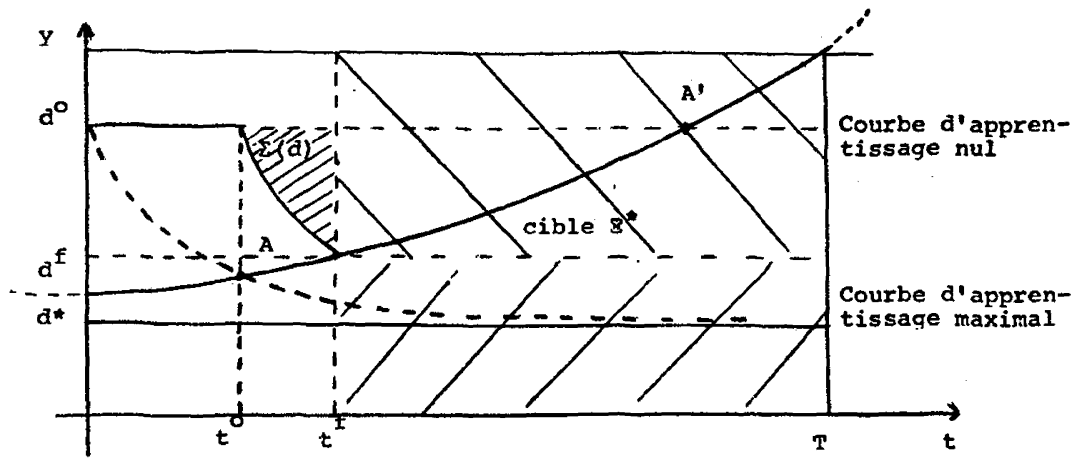

Figure 5

L'arc ouvert $A A^{\prime}$ de $1^{\prime}$ hyperbole $\Xi^{*}$ est la partie utile de la cible. Les points situés sur cet arc peuvent définir un accord possible, acceptable par $E$.

Il est intéressant de remarquer que $E$ construit cette cible sans avoir complètement déterminé la stratégie de $S$. $E$ sait seulement que la "commande » adoptée par $S$ pour réduire ses revendications sera de type «Bang-Bang ".

La cible $\Xi^{*}$ peut s'interpréter comme étant une stratégie de $E$. En effet, il s'agit d'un plan d'action définissant la décision de $E$ en fonction de l'état $(t, d)$ du système. L'ensemble des stratégies possibles pour $E$ correspond à un ensemble $\zeta_{E}$ de fonctions $\theta:(t, d) \rightarrow \theta(t, d) \forall t \in[0, T], \forall d \in\left[d^{*}, y\right]$.

Une stratégie $\theta \in \zeta_{E}$ peut se «lire»: «Accepter l'accord $\left(t^{f}, d^{f}\right) \operatorname{si} \theta\left(t^{f}\right.$, $\left.d^{f}\right)=0$.

Pour définir la stratégie $\theta^{*}$ donnée en (9), $E$ suppose que $S$ a adopté aussi une stratégie sous forme d'une fonction $s:(t, d) \rightarrow u=s(t, d) \in[0$, $\tau]$ définissant le taux de concession à partir de l'état du système. Comme $S$ est seul à contrôler l'histoire de la négociation et que cette histoire influe sur sa fonction d'utilité, $E$ peut en déduire que $S$ cherchera en tout état de cause à rendre la négociation moins préjudiciable et donc sera intransigeant sur une partie 
de l'ensemble des états $(t, d)$ possibles et au contraire conciliant sur un sous ensemble de l'ensemble complémentaire de cette partie. Pour pouvoir définir la cible $\Xi^{*}, E$ est obligé de supposer que $S$ a une stratégie de concession maximale au voisinage de l'arc ouvert $A A^{\prime}$. Pour une demande initiale $d^{\circ}$ fixée, cette condition va être remplie. Au point $A, E$ connaît encore la valeur de $\dot{d}$ cependant qu'au point $A^{\prime}$ on ne peut rien dire. En effet, s'il est extrêmement coûteux pour $S$ de s'éloigner de $d^{\circ}, S$ aura une politique d'intransigeance absolue et $E$ aurait avantage à accepter l'accord en $\left(0, d^{\circ}\right)$. Nous ne considérons donc pour l'instant que l'arc ouvert $A A^{\prime}$ que nous avons appelé la partie utile de la cible.

\subsection{Construction d'une stratégie optimale pour $S$}

La cible $\Xi^{*}$ a été déterminée par le souci de chaque joueur d'obtenir un gain maximal.

Étant donné $d^{\circ}, S$ va chercher un point sur l'arc $A A^{\prime}$ de la cible, qui rende maximal son gain.

En tout point $\left(t^{f}, d^{f}\right)$ de $A A^{\prime}$, nous pouvons calculer le gain de $S$ résultant de la négociation ayant débuté en $t=0$ avec une revendication $d^{\circ}$.

Puisqu'on connaît la forme de "L'histoire de la négociation ", on peut calculer en remontant le temps la valeur de la fonctionnelle de gain, et on obtient :

$$
\begin{aligned}
g_{S} & \triangleq V_{s}\left(t^{f}, d^{f}\right) \\
& =d^{f}\left(T-t^{f}\right)+\alpha \int_{0}^{t^{\prime}}\left[d(t)-d^{\circ}\right] d t
\end{aligned}
$$

Or, d'après (7) :

$$
\int_{0}^{t^{t}}\left[d(t)-d^{\circ}\right] d t=\int_{t^{c}}^{t^{t}}\left[d(t)-d^{\circ}\right] d t
$$

d'autre part, puisque, entre les instants $t^{C}$ et $t^{\mathcal{S}}$ :

$$
\dot{d}(t)=-\tau\left(d(t)-d^{*}\right)
$$

on en déduit que :

$$
d(t)=K \mathrm{e}^{-\tau t}+d^{*} \quad \text { si } \quad t^{c} \leqslant t \leqslant t^{f} .
$$

$K$ est une constante, et

$$
\begin{aligned}
& d^{f}=K \mathrm{e}^{-\tau t^{f}}+d^{*} \\
& d\left(t^{c}\right)=d^{\circ}=K \mathrm{e}^{-\tau t^{c}}+d^{*}
\end{aligned}
$$

$\mathbf{n}^{\circ} \mathrm{V}-1,1972$. 
d'où :

ce qui permet d'écrire :

$$
\begin{aligned}
t^{f} & =-\frac{1}{\tau} \log \frac{d^{f}-d^{*}}{K} \\
t^{c} & =-\frac{1}{\tau} \log \frac{d^{f}-d^{*}}{d^{\circ}-d^{*}}
\end{aligned}
$$

$$
\begin{aligned}
\int_{t^{\sigma}}^{t^{t}} & {\left[d(t)-d^{\circ}\right] d t=\int_{t^{\sigma}}^{t^{t}}\left(K \mathrm{e}^{-\tau t}+d^{*}-d^{\circ}\right) d t } \\
& =\left(d^{*}-d^{\circ}\right)\left(t^{f}-t^{c}\right)-\frac{1}{\tau}\left(K \mathrm{e}^{-\tau t^{f}}-K \mathrm{e}^{-\tau t^{c}}\right) \\
& =-\frac{1}{\tau}\left(d^{*}-d^{\circ}\right) \log \frac{d^{f}-d^{*}}{d^{\circ}-d^{*}}-\frac{1}{\tau}\left(d^{f}-d^{\circ}\right)
\end{aligned}
$$

d'où :

$$
g_{s}=d^{f}\left(T-t^{f}\right)-\frac{\alpha}{\tau}\left(d^{*}-d^{\circ}\right) \log \frac{d^{f}-d^{*}}{d^{\circ}-d^{*}}-\frac{\alpha}{\tau}\left(d^{f}-d^{\circ}\right)
$$

ou encore, en posant :

$$
\begin{cases}A=\frac{\alpha}{\tau}\left(d^{\circ}-d^{*}\right) \log \left(d^{\circ}-d^{*}\right) & A \geqslant 0 \\ B=\frac{\alpha}{\tau}\left(d^{\circ}-d^{*}\right) & B \geqslant 0\end{cases}
$$

(10) $g_{s} \triangleq V_{S}\left(t^{f}, d^{f}\right)$

$$
=-A+B \log \left(d^{f}-d^{*}\right)+\frac{\alpha}{\tau}\left(d^{\circ}-d^{f}\right)+d^{f}\left(T-t^{f}\right) .
$$

S'il existe un point «intérieur » à l'arc $A A^{\prime}$ rendant $V_{s}\left(t^{f}, d^{f}\right)$ maximal, nous devrons avoir en ce point les conditions :

$$
\left\{\begin{array}{l}
\frac{\partial V_{s}}{\partial t^{f}}+\nu \frac{\partial \theta^{*}}{\partial t^{f}}=\frac{\partial V_{s}}{\partial d^{f}}+\nu \frac{\partial \theta^{*}}{\partial d^{f}}=0 \\
\theta^{*}\left(t^{f}, d^{f}\right)=0
\end{array}\right.
$$

où. $v$ est un multiplicateur de Lagrange associé par $S$ à la cible. Ces conditions s'écrivent aussi :

(11)

$$
-d^{f}-v=0
$$

$$
\text { (12) } \frac{B}{d^{f}-d^{*}}-\frac{\alpha}{\tau}+T-t_{f}+\nu\left[\frac{1}{\tau\left(d^{f}-d^{*}\right)}+\frac{y-d^{f}}{\tau\left(d^{f}-d^{*}\right)^{2}}\right]=0
$$




$$
T-t^{f}-\frac{y-d^{f}}{\tau\left(d^{f}-d^{*}\right)}=0
$$

Remarquons que la relation (11) définit simplement le multiplicateur de Lagrange $\nu$ comme égal à la variation marginale de $g_{s}$ quand il faut attendre un peu plus longtemps pour atteindre l'accord.

De (11)-(13), on tire la relation :

$$
\frac{B}{d^{f}-d^{*}}-\frac{\alpha}{\tau}+\frac{y-d^{f}}{\tau\left(d^{f}-d^{*}\right)}-d^{f}\left[\frac{y-d^{*}}{\tau\left(d^{f}-d^{*}\right)^{2}}\right]=0
$$

soit :

$$
\tau B\left(d^{f}-d^{*}\right)-\alpha\left(d^{f}-d^{*}\right)^{2}+\left(y-d^{f}\right)\left(d^{f}-d^{*}\right)-d^{f}\left(y-d^{*}\right)=0
$$

ce qui s'écrit :

$$
\tau B\left(d^{f}-d^{*}\right)-\alpha\left(d^{f}-d^{*}\right)^{2}-\left(d^{f}-d^{*}\right)^{2}+d^{*}\left(d^{*}-y\right)=0
$$

ou encore :

$$
\text { (14) }-(1+\alpha)\left(d^{f}-d^{*}\right)^{2}+\tau B\left(d^{f}-d^{*}\right)+d^{*}\left(d^{*}-y\right)=0
$$

qui est une équation du second degré en $\left(d^{f}-d^{*}\right)$, déterminant $d^{f}$.

L'existence et le nombre des racines de (14) dépendront du signe de l'expression :

$$
\alpha^{2}\left(d^{\circ}-d^{*}\right)^{2}-4(1+\alpha) d^{*}\left(y-d^{*}\right)
$$

Si on a :

$$
\frac{\alpha^{2}}{1+\alpha}<\frac{4\left(y-d^{*}\right) d^{*}}{\left(d^{\circ}-d^{*}\right)^{2}}
$$

il n'existe pas de point intérieur à la partie utile de la cible maximisant $g_{s}$.

Le second membre de (16) est positif; nous voyons donc que l'inégalité (16) sera vérifiée pour $\alpha$ suffisamment grand. Cela revient à dire que, si le coût des concessions augmente, $S$ ne pourra jamais concéder et donc ne pourra pas atteindre la partie utile de la cible. Nous reviendrons plus tard sur ce type de comportement.

Si on a :

$$
\frac{\alpha^{2}}{1+\alpha}>\frac{4\left(y-d^{*}\right) d^{*}}{\left(d^{\circ}-d^{*}\right)^{2}}
$$

alors, il existe deux racines positives de (14) données par :

$$
d^{f}-d^{*}=\frac{B \tau \pm \sqrt{B^{2} \tau^{2}-4(1+\alpha) d^{*}\left(y-d^{*}\right)}}{2(1+\alpha)}
$$

$\mathrm{n}^{\circ} \mathrm{V}-1,1972$. 
Ces racines sont toutes deux intérieures à l'intervalle $\left(d^{\circ}, d^{*}\right)$.

En effet, on montre facilement que, si $d^{f}$ vérifie l'équation (14), on a toujours : $d^{f}<d^{\circ}$.

On obtient donc deux points extrémaux $\left(t_{1}^{f}, d_{1}^{f}\right)$ et $\left(t_{2}^{f}, d_{2}^{f}\right)$ où on a supposé : $t_{1}^{f}<t_{2}^{f}$.

Remarquons que le gain de $E$ est supérieur en $\left(t_{1}^{f}, d_{1}^{f}\right)$ mais que seules des considérations relatives au gain de $S$ déterminent le point d'accord sur la cible; ce sera celui des deux points extrémaux correspondant au maximum de $V_{S}\left(t^{f}, d^{f}\right)$.

Ce point d'accord que nous venons d'obtenir a les propriétés caractéristiques d'un équilibre de Nash. Le gain de chaque joueur est en effet rendu maximal étant donné le comportement de l'adversaire.

La notion d'équilibre de Nash a souvent été contestée comme critère d'optimalité ayant une valeur opérationnelle. Dans ce modèle, il semble cependant que l'équilibre de Nash soit une solution «naturelle » de ce type de jeu. En effet, il y a une hiérarchie dans les joueurs puisque le syndicat « joue le premier » en concédant, cependant, que l'entreprise n'intervient que pour mettre fin au jeu.

\subsection{Intransigeance de $S$}

Supposons que $\alpha$ soit suffisamment grand pour que le gain de $S$ aille en croissant quand le point d'accord décrit l'arc $A A^{\prime}$. Le point $A^{\prime}$ ne correspond pas à un équilibre de Nash. En effet, pour $E$, étant donnée l'intransigeance de $S$, le mieux est d'accepter $d^{\circ}$ dès l'instant initial. Remarquons cependant que $E$ peut ne pas connaître précisément la valeur de $\alpha$ et espérer une concession de $S$. Quand le point $A^{\prime}$ est atteint, $E$ sait que $S$ est intransigeant et accepte l'accord.

\subsection{Recherche des points efficaces}

Le syndicat effectue sa demande $d^{\circ}$. Un point efficace sera alors un point $\left(t_{E}^{f}, d_{E}^{f}\right) \in K_{d}$ o tel qu'il n'existe pas $\left(t^{f}, d^{f}\right)$ vérifiant les conditions :

$$
\begin{aligned}
& g_{S}=V_{S}\left(t^{f}, d^{f}\right) \geqslant V_{S}\left(t_{E}^{f}, d_{E}^{f}\right) \\
& g_{E}=V_{E}\left(t^{f}, d^{f}\right) \geqslant V_{E}\left(t_{E}^{f}, d_{E}^{f}\right)
\end{aligned}
$$

une des conditions au moins étant une inégalité stricte.

Une courbe d'indifférence pour $E$ sera l'ensemble des points $\left(t^{f}, d^{f}\right)$ tels que :

$$
\left(T-t^{f}\right)\left(y-d^{f}\right)=K_{1}
$$


c'est-à-dire :

$$
d^{f}=y+\frac{K_{1}}{t^{f}-T}
$$

De la même façon, une courbe d'indifférence pour $S$ sera l'ensemble des points $\left(t^{f}, d^{f}\right)$ tels que :

$$
\left(\dot{T} \dot{-} t^{f}\right) d^{f}-\alpha \int_{\sigma^{o}}^{t^{\prime}}\left[d^{\circ}-\dot{d}(t)\right] d t=K_{2}
$$

soit, d'après (10) :

$$
d^{f}\left(T-t^{f}\right)-A+B \log \left(d^{f}-d^{*}\right)+\frac{\alpha}{\tau}\left(d^{\circ}-d^{f}\right)=K_{2}
$$

ou encore :

$$
t^{f}=T-\frac{\alpha}{\tau}-\frac{K_{2}+A-\frac{\alpha}{\tau} d^{\circ}}{d^{f}}+B \frac{\log \left(d^{f}-d^{*}\right)}{d^{f}} .
$$

Considérons le point $A$, point d'intersection de la cible' et de la courbe d'apprentissage maximal de $S$. Les coordonnées $\left(t_{A}^{f}, d_{A}^{f}\right)$ de ce point vérifient l'équation de la cible, soit :

$$
T-t_{A}^{f}-\frac{y-d_{A}^{f}}{\tau\left(d_{A}^{f}-d^{*}\right)}=0
$$

c'est-à-dire :

$$
d_{A}^{f}=d^{*}+\frac{y-d^{*}}{1+\tau\left(T-t_{A}^{f}\right)}
$$

et l'équation de la courbe d'apprentissage maximal, soit :

$$
d_{A}^{f}=\left(d^{\circ}-d^{*}\right) \mathrm{e}^{-\tau t_{A}^{f}}+d^{*}
$$

D'autre part, la courbe d'indifférence de $E$ passant par $A$ est telle que :

$$
d^{f}=y+\frac{K_{A}}{t^{f}-T} \quad \mathrm{~d} \text { 'où : } \quad K_{A}=\left(d_{A}^{f}-y\right)\left(t_{A}^{f}-T\right)
$$

ou, d'après (20): $K_{A}=\frac{-\tau\left(d^{*}-y\right)\left(T-t_{A}^{f}\right)^{2}}{1+\tau\left(T-t_{A}^{f}\right)} \cdot$

Les tangentes en $A$ à cette courbe d'indifférence et à la courbe d'apprentissage maximal ont pour pente :

$$
\pi=-\frac{K_{A}}{\left(t_{A}^{f}-T\right)^{2}}=\frac{\tau\left(d^{*}-y\right)}{1+\tau\left(T-t_{A}^{f}\right)}
$$

no V-1, 1972. 
et $\pi^{\prime}=-\tau\left(d_{A}^{f}-d^{*}\right)$ respectivement

or, d'après (20),

$$
\pi^{\prime}=-\tau \frac{\left(y-d^{*}\right)}{1+\tau\left(T-t_{A}^{f}\right)}=\pi .
$$

En $A$, la courbe d'indifférence de $E$ passant par $A$ et la courbe d'apprentissage maximal de $S$ sont donc tangentes. Étant donnée la forme des courbes d'indifférence de $E$, on a :

$$
\begin{aligned}
\underset{K_{d \circ}}{\operatorname{Max}_{i}} & V_{E}\left(t^{f}, d^{f}\right)=\underset{K_{d \circ}}{\operatorname{Max}}\left(T-t^{f}\right)\left(y-d^{f}\right) \\
& =\left(T-t_{A}^{f}\right)\left(y-d_{A}^{f}\right)=V_{E}\left(t_{A}^{f}, d_{A}^{f}\right) .
\end{aligned}
$$

D'autre part, l'étude des courbes d'indifférence de $E$ et $S$ montre que l'ensemble $\mathcal{C}$ des points efficaces est l'ensemble des points de la courbe d'apprentissage maximale compris entre le point $\left(0, d^{\circ}\right)$ et le point $A$. (Il est facile de le voir en étudiant le maximum du gain de $S$ (resp. $E$ ), étant donné un gain de $E$ (resp. $S$ ) constant.)

Une fois que le syndicat a effectué sa demande $d^{\circ}$, les théories classiques de la négociation conduiraient alors à un point d'accord sur $\mathcal{C}$.

Un tel point n'est pas acceptable étant données les hypothèses du modèle concernant le processus de négociation. En effet, si le syndicat adopte une politique de conciliation, c'est-à-dire adopte un taux de concession maximal, l'entreprise peut refuser son accord le plus longtemps possible.

Un point d'accord sur $\mathcal{C}$ n'est donc pas stable, s'il diffère du point $A$.

\section{Conclusion de la première partie}

Le modèle que nous venons d'étudier présente de trop nombreuses imperfections pour pouvoir prétendre être opérationnel. Cependant nous avons pu montrer que la considération du processus de négociations dans son aspect essentiellement dynamique permet de faire apparaître une solution bien définie et ayant un certain sens économique à ce jeu.

La théorie des jeux a un aspect normatif qui l'a desservie auprès des économistes appliqués. Nous croyons cependant qu'un grand nombre des difficultés actuelles rencontrées dans l'application de la théorie des jeux aux problèmes concrets provient du manque d'intérêt porté jusqu'à ces dernières années à l'étude de la valeur du temps dans les processus de négociation. Les travaux de Cross, Case et Contini constituent une base solide pour donner un élan nouveau à la théorie des jeux de négociation.

Bien que nous ayons proposé une solution à ce problème de négociation il nous semble important de revenir sur la structure de ce type de jeu de façon à en généraliser les possibilités d'application. 


\section{2e PARTIE : EQULIBRE DE NASH DANS UN JEU SEMI-DIFFERENTIEL}

\section{Introduction}

Dans la première partie de cette étude nous avons présenté un modèle de négociations industrielles que nous avons traité par une méthode adaptée à sa structure particulière. Dans cette seconde partie nous donnerons une définition générale de ce type de jeu que nous appelerons jeu semi-différentiel. Dans un jeu différentiel, plusieurs joueurs pilotent de façon conflictuelle un certain système. Dans un jeu semi-différentiel, seul un joueur pilote le système cependant que le second joueur n'a qu'une seule décision à prendre, celle de mettre fin au jeu.

La terminologie que nous adopterons est en grande partie celle que A. Blaquière et G. Leitmann ont utilisée dans leurs travaux ([3], [4], [13]). Nous établirons un résultat donnant une condition nécessaire d'optimalité propre à ce type de jeu que nous appliquerons au modèle déjà présenté.

\section{Equation, d'état, gains, stratégies}

Nous considèrerons un système dynamique régi par l'équation d'état :

$$
\dot{x}=f(x, u)
$$

où $x \in E^{n}$ est la variable d'état, $u \in E^{m}$ la variable de commande. Nous supposons que la n-ième composante de la variable

$$
x \triangleq\left(x_{1}, x_{2}, \ldots, x_{n}\right) \in E^{n}
$$

représente le temps $\left(x_{n}=t\right)$.

Soient $G$ et $U$ des domaines de $E^{n}$ et $E^{m}$ respectivement, nous supposons que les composantes de la fonction $f$,

$$
f \triangleq\left(f_{1}, f_{2}, \ldots, f_{n}\right) \quad ; \quad f_{n}(x, u) \equiv 1
$$

sont de classe $C^{1}$ sur $G \times U$.

La variable de commande $u$ est à la disposition d'un joueur $J_{1}$.

Soit :

$$
x_{0} \in \mathbf{R}
$$

une variable réelle repérant un gain accumulé par ce joueur. $n^{\circ}$ V-1, 1972. 
L'équation d'accumulation du gain de $J_{1}$ est donnée par :

$$
\dot{x}_{0}=f_{0}(x, u)
$$

où $f_{0}$ est aussi de classe $C^{1}$ sur $G \times U$.

Soit $x^{\circ}$ un état initial, on a :

$$
x_{n}^{\circ}=t_{0}
$$

A cet état initial et à une commande

$$
\tilde{u}: t \rightarrow u=\tilde{u}(t) \in U \quad, \quad t_{0} \leqslant t \leqslant t_{1}
$$

mesurable sur $\left[t_{0}, t_{1}\right]$ correspond une solution unique de l'équation (1):

$$
\tilde{x}: t \rightarrow x=\tilde{x}(t) \in E^{n} \quad, \quad \tilde{x}\left(t_{0}\right)=x^{\circ} .
$$

$\mathrm{Si}, \forall t \in\left[t_{0}, t_{1}\right]$, on a :

$$
\tilde{x}(t) \in G
$$

on dit que $\tilde{x}$ est une réponse admissible du système, engendrée à partir de $x^{\circ}$, par la commande $\tilde{u}$.

Alors que le joueur $J_{1}$ pilote le système un second joueur, $J_{2}$, définit seul l'instant terminal $t_{f}$. On aura évidemment :

$$
t_{f} \geqslant t_{0}
$$

Considérons enfin deux fonctions :

$$
\Phi_{i}: G \rightarrow R ; i=1,2
$$

respectivement de classe $C^{1}$ et $C^{2}$ sur $G$, appelées fonctions de gain terminal de chaque joueur $J_{i} ; i=1,2$.

Si $\tilde{x}$ est une réponse admissible engendrée à partir de $x^{\circ}$ par la commande $\tilde{u}$, définie sur l'intervalle $\left[t_{0}, t_{1}\right]$ et si l'instant final est $t_{f} \in\left[t_{0}, t_{1}\right]$ alors le joueur $J_{1}$ obtient un gain :

$$
G_{1}\left(x^{\circ}, t_{f}, \tilde{x}, \tilde{u}\right) \triangleq \Phi_{1}\left(\tilde{x}\left(t_{f}\right)\right)+\int_{t_{0}}^{t_{f}} f_{0}(\tilde{x}(t), \tilde{u}(t)) \mathrm{d} t
$$

et le joueur $J_{2}$ obtient un gain :

$$
G_{2}\left(x^{\circ}, t_{f}, \tilde{x}, \tilde{u}\right) \triangleq \Phi_{2}\left(\tilde{x}\left(t_{f}\right)\right) .
$$


Étant donné que le gain de chaque joueur est affecté par la décision de l'adversaire et que ce problème est de nature dynamique, chaque joueur doit adopter une stratégie liant sa décision à l'état du système.

$\zeta_{1} \triangleq\left\{s_{1}\right\}$ est l'ensemble des stratégies du joueur $J_{1}$. Les $s_{1}$ sont des fonctions sur un domaine $X \subset G$ :

$$
s_{1}: X \rightarrow U, \quad x \rightarrow u=s_{1}(x)
$$

qui sont soumises à des contraintes du type général suivant :

$$
s_{1}(x) \in K_{1}(x) \subset U \quad \forall x \in X .
$$

Ces stratégies satisfont aux règles de composition habituelles données dans [3] par exemple. $\zeta_{2} \triangleq\left\{\theta_{2}\right\}$ est l'ensemble des stratégies du joueur $J_{2}$. Les $\theta_{2}$ sont des fonctions définies sur le domaine $G$, à valeurs réelles et telles que les ensembles :

$$
\Xi_{2}=\theta_{2}^{-1}(0) \triangleq\left\{x \in G: \theta_{2}(x)=0\right\}
$$

soient non vides et fermés. $\Xi_{2}$ joue alors le rôle d'une cible. Quand, au cours d'une réponse, l'état atteint la cible, le jeu est terminé.

\section{Couple stratégique jouable. Equilibre de Nash}

Soit $\left(s_{1}, \theta_{2}\right) \in \zeta_{1} \times \zeta_{2}$ un couple stratégique où $s_{1}$ est une fonction définie sur $X$.

Définition 1: «Le couple $\left(s_{1}, \theta_{2}\right)$ est jouable en $x^{i} \in X$ s'il existe une fonction

$$
\tilde{x}: t \rightarrow x=\tilde{x}(t) \in X \forall t \in\left[t_{i}, t_{j}\right] \quad ; \quad t_{i}=x_{n}^{i}
$$

vérifiant, presque partout sur $\left[t_{i}, t_{j}\right]$, l'équation différentielle :

$$
\frac{\mathrm{d} \tilde{x}(t)}{\mathrm{d} t}=f\left(\tilde{x}(t) \quad, \quad s_{1}(\tilde{x}(t))\right)
$$

et la condition initiale :

$$
\tilde{x}\left(t_{i}\right)=x^{2}
$$

et telle que pour un instant $t_{f} \in\left[t_{i}, t_{j}\right]$ on ait :

$$
\begin{aligned}
& \tilde{x}\left(t_{f}\right) \in \Xi_{2} \\
& \tilde{x}(t) \in G-\Xi_{2} \quad \text { si } \quad t \in\left[t_{i}, t_{f}\right) .
\end{aligned}
$$

Nous dirons alors que $\tilde{x}$ est une réponse complète de (7) engendrée par $s_{1}$. $n^{\circ} \mathrm{V}-1,1972$. 
Nous noterons :

$$
V_{1}\left(x^{i}, \Xi_{2}, s_{1}\right) \triangleq \Phi_{1}\left(\tilde{x}\left(t_{f}\right)\right)+\int_{t_{i}}^{t_{f}} f_{0}\left(\tilde{x}(t), s_{1}(\tilde{x}(t))\right) \mathrm{d} t
$$

le gain du joueur $J_{1}$ obtenu le long de la réponse $\tilde{x}$ et engendré par le couple stratégique $\left(s_{1}, \theta_{2}\right)$. (Nous supposerons que l'intégrale est définie pour tout $s_{1} \in \zeta_{1}$.)

De même façon nous noterons :

$$
V_{2}\left(x^{i}, \Xi_{2}, s_{1}\right) \triangleq \Phi_{2}\left(\tilde{x}\left(t_{f}\right)\right)
$$

le gain du joueur $J_{2}$ engendré par le couple $\left(s_{1}, \theta_{2}\right)$. Remarquons que les expressions (8) et (9) sont imprécises puisque, a priori, plusieurs réponses peuvent être engendrées par une même stratégie (cf. [3]). Néanmoins nous conserverons ces notations pratiques pour définir la notion d'optimalité au sens de Nash.

Définition 2 : « Le couple stratégique $\left(s_{1}^{*}, \Xi_{2}^{*}\right)$ est un équilibre sur $X$ si :

(i) l'ensemble $X^{*} \subset X$ des points où $\left(s_{1}^{*}, \Xi_{2}^{*}\right)$ est jouable est non vide.

(ii) $\forall x^{i} \in X^{*}, \forall \theta_{2} \in \zeta_{2}$ tel que $\left(s_{1}^{*}, \theta_{2}\right)$ soit jouable en $x^{i}$ on a :

$$
V_{2}\left(x^{i}, \Xi_{2}, s_{1}^{*}\right) \leqslant V_{2}\left(x^{i}, \Xi_{2}^{*}, s_{1}^{*}\right)
$$

(iii) $\forall s_{1} \in \zeta_{1}$ tel que $\left(s_{1}, \theta_{2}^{*}\right)$ soit jouable en $x^{i}$ on a :

$$
V_{1}\left(x^{i}, \Xi_{2}^{*}, s_{1}\right) \leqslant V_{1}\left(x^{i}, \Xi_{2}^{*}, s_{1}^{*}\right) .
$$

RemarQue : Les conditions (ii) et (iii) font que les gains $V_{j}\left(x^{i}, \Xi_{2}^{*}, s_{1}^{*}\right)$, $=1,2$, sont uniquement définis.

IV. Une condition nécessaire concernant la cible $\Xi_{2}^{*}$.

Soit $\left(s_{1}^{*}, \theta_{2}^{*}\right)$ un équilibre sur $X$ et $x^{i} \in X^{*}$, un état initial donné. Soit :

$$
\tilde{x}^{*}: t \rightarrow x=\tilde{x}^{*}(t) \quad \forall t \in\left[t_{i}, t_{j}\right]
$$

une réponse complète de (7), engendrée par $s_{1}^{*}$. On a

$$
\begin{aligned}
& \tilde{x}^{*}\left(t_{i}\right)=x^{i} \\
& \exists t_{f} \in\left[t_{i}, t_{j}\right] \text { tel que } \tilde{x}^{*}\left(t_{f}\right) \in \Xi_{2}^{*} \text { et } \\
& x^{*}(t) \notin \Xi_{2}^{*} \quad \forall t \in\left[t_{i}, t_{f}\right) .
\end{aligned}
$$

Nous ferons un certain nombre d'hypothèses sur la nature des stratégies des deux joueurs. 
Hypothèse 1 : "Il existe un voisinage $\Delta\left(\tilde{x}^{*}\left(t_{f}\right)\right)$ de $\tilde{x}^{*}\left(t_{f}\right)$ dans $X$ dans lequel $s_{1}^{*}$ est continue et $\theta_{2}^{*}$ est de classe $C^{1}$.»

Hypothèse 2: "Il existe un voisinage $\Delta\left(\tilde{x}^{*}\left(t_{f}\right)\right)$ tel que pour tout $x \in \Delta\left(\tilde{x}^{*}\left(t_{f}\right)\right) \cap \Xi_{2}^{*}$ on ait :

$$
\operatorname{Grad} \theta_{2}^{*}(x) \cdot f\left(x, s_{1}^{*}(x)\right) \neq 0 \cdot »
$$

Hypothèse 3 : "L'ensemble de stratégies $\zeta_{2}$ est tel que, dans un voisinage $\Delta\left(\tilde{x}^{*}\left(t_{f}\right)\right)$, pour tout point $x^{f} \in \Delta\left(\tilde{x}^{*}\left(t_{f}\right)\right)$ il existe une cible $\Xi_{2}$ contenant ce point :

$$
x^{f} \in \Xi_{2} \cdot »
$$

Ces hypothèses jouent les rôles suivants :

L'hypothèse 1 nous permet de considérer que l'instant terminal $t_{f}$ est intérieur au segment $\left[t_{i}, t_{j}\right]$ :

$$
t_{f} \in\left(t_{i}, t_{j}\right)
$$

L'hypothèse 2 nous permet d'éviter le cas où la trajectoire représentée par $\tilde{x}^{*}: t \rightarrow x=\tilde{x}^{*}(t) \quad \forall t \in\left[t_{i}, t^{f}\right]$ atteint $\Xi_{2}^{*}$ tangentiellement.

L'hypothèse 3 enfin nous permet de supposer que le joueur $J_{2}$ peut interrompre le jeu en n'importe quel point de $\Delta\left(\tilde{x}^{*}\left(t_{f}\right)\right)$.

Nous sommes alors en mesure d'établir le résultat suivant :

Proposition : Si les hypothèses 1-3 sont vérifiées alors il existe un voisinage $\Delta\left(\tilde{x}^{*}\left(t_{f}\right)\right)$ tel que l'on ait :

$$
\begin{gathered}
\Xi_{2}^{*} \cap \Delta\left(\tilde{x}^{*}\left(t_{f}\right)\right) \subset\left\{x \in \Delta\left(\tilde{x}^{*}\left(t_{f}\right)\right):\right. \\
\left.\operatorname{grad} \Phi_{2}(x) \cdot f\left(x, s_{1}^{*}(x)\right)=0\right\} .
\end{gathered}
$$

Démonstration : Soit $\Delta\left(x^{*}\left(t_{f}\right)\right)$ un voisinage de $x^{*}\left(t_{f}\right)$ dans $X$ tel que les trois hypothèses soient vérifiées. Soit un point :.

$$
\cdot x^{f} \in \Xi_{2}^{*} \cap \Delta\left(\tilde{x}^{*}\left(t_{f}\right)\right) \text {. }
$$

D'après l'hypothèse 1 il existe une solution $\tilde{x}($.$) de l'équation. (7), engen-$ drée par $s_{1}^{*}$, définie sur un intervalle $\left[t_{l}, t_{k}\right]$ et telle que l'on ait :

$$
\begin{aligned}
& \tau_{f} \triangleq x_{n}^{f} \in\left(t_{l}, t_{k}\right) \\
& \tilde{x}\left(\tau_{f}\right)=x^{f} .
\end{aligned}
$$

$n^{\circ}$ V-1, 1972. 
D'après l'hypothèse 2 on peut choisir $t_{l}$ de telle façon que l'on ait pour tout instant $t \in\left[t_{l}, \tau_{f}\right)$ la relation :

$$
x(t) \in \Delta\left(\tilde{x}^{*}\left(t_{f}\right)\right)-\Xi_{2}^{*}
$$

D'après l'hypothèse 3 , pour tout $\tilde{x}(t), t \in\left[t_{l}, t_{k}\right]$, il existe une stratégie $\theta_{2} \in \zeta_{2}$ telle que :

$$
\tilde{x}(t) \in \Xi_{2} \text {. }
$$

Comme le couple $\left(s_{1}^{*}, \Xi_{2}^{*}\right)$ est un équilibre sur $X$, d'après la définition 2 nous devrons avoir :

$$
\begin{aligned}
V_{2}\left(\tilde{x}\left(t_{l}\right), \Xi_{2}^{*}, s_{1}^{*}\right) & =\Phi_{2}\left(x^{f}\right) \geqslant \\
V_{2}\left(\tilde{x}\left(t_{l}\right), \Xi_{2}, s_{1}^{*}\right) & =\Phi_{2}(\tilde{x}(t)) .
\end{aligned}
$$

pour tout instant $t \in\left(t_{l}, t_{k}\right)$.

En dérivant $\Phi_{2}(\tilde{x}(\cdot))$, par rapport à $t$, à l'instant $\tau_{f} \in\left(t_{l}, t_{k}\right)$ nous obtenons la condition nécessaire :

$$
\operatorname{grad} \Phi_{.2}\left(x^{f}\right) \cdot f\left(x^{f}, s_{1}^{*}\left(x^{f}\right)\right)=0
$$

qui établit la proposition.

V. Conditions nécessaires d'optimalité pour le joueur $J_{1}$

D'après la définition 2 , la stratégie $s_{1}^{*}$ est une stratégie de commande optimale, relativement à la fonctionnelle de gain du joueur $J_{1}$, étant donnée la cible $\Xi_{2}^{*}$, sur l'ensemble $X^{*}$. On peut appliquer alors les résultats classiques de la théorie de la commande optimale pour obtenir des conditions nécessaires d'optimalité sous la forme d'un principe du maximun ou d'une équation de type programmation dynamique.

\section{Application au modèle de négociations}

Considérons la variable d'état $x \in \mathbf{R}$, la variable de commande $u \in[0, \tau] \subset \mathbf{R}$, et l'équation d'état :

$$
\dot{x}=-u\left(x-d^{*}\right)
$$

ainsi que l'équation d'accumulation de gain de $J_{1}$ :

$$
\dot{x}_{0}=-\alpha\left(d^{\circ}-x\right)
$$

où $d^{\circ}$ et $d^{*}$ sont des nombres réels donnés vérifiant :

$$
d^{\circ}-d^{*}>0 .
$$


Soient d'autre part les fonctions de gain terminal :

$$
\begin{aligned}
& \Phi_{1}(x, t)=x(T-t) \\
& \Phi_{2}(x, t)=(y-x)(T-t)
\end{aligned}
$$

où $y$ et $T$ sont des nombres réels vérifiant :

$$
\left\{\begin{array}{l}
y-d^{\circ} \geqslant 0 \\
T-t \geqslant 0
\end{array}\right.
$$

L'état initial du système est :

$$
x^{\circ}=d^{\circ} \quad t_{0}=0 .
$$

Soit $\left(s_{1}^{*}, \theta_{2}^{*}\right)$ un équilibre de Nash sur un domaine $X$ engendrant à partir de $\left(d_{\mathbb{Z}}^{\circ}, 0\right)$ une réponse $\tilde{x}^{*}: t \rightarrow x=\tilde{x}^{*}(t) \forall t \in\left[0, t_{l}\right]$ telle que $\tilde{x}^{*}\left(t_{f}\right)$ soit contenu dans la cible $\Xi_{2}^{*}$ à un instant $t_{f} \in\left(0, t_{l}\right)$.

Supposons que les hypothèses 1-3 soient vérifiées et que le long de la réponse $\tilde{x}^{*}$ on puisse utiliser le principe du maximum. Le long de cette réponse considérons une fonction :

$$
\tilde{\lambda}: t \rightarrow \lambda=\tilde{\lambda}(t) \in \mathbf{R} \quad \forall ' t \in\left[0, t_{f}\right]
$$

vérifiant l'équation variationnelle adjointe :

$$
\dot{\lambda}=-\alpha+u \lambda
$$

et les conditions de transversalité (cf. [5] ou [13]).

$$
\left.\tilde{\lambda}\left(t_{f}\right)=\frac{\partial \Phi_{1}}{\partial x}+\nu \frac{\partial \theta_{2}^{*}}{\partial x}\right]\left.\right|_{\substack{x=\tilde{x} *(t) \\ t=t_{f}}}
$$

$$
\left.0=\frac{\partial \Phi_{1}}{\partial t}+\nu \frac{\partial \theta_{2}^{*}}{\partial t}-\tilde{\lambda}\left(t_{f}\right) s_{1}^{*}(x)\left(x-d^{*}\right)-\alpha\left(d^{\circ}-x\right)\right] \mid \begin{aligned}
& x=\tilde{x}^{*}\left(t_{j}\right) \\
& t=t_{i j}
\end{aligned}
$$

où $v$ est un multiplicateur de Lagrange associé à la cible..

Si nous formons l'hamiltonien :

$$
H(u)=-\alpha\left(d^{\circ}-x\right)-\tilde{\lambda}(t) u\left(\tilde{x}^{*}(t)-d^{*}\right)
$$

nous devrons alors avoir nécessairement :

$$
H\left(s_{1}^{*}\left(\tilde{x}^{*}(t)\right)\right)=\underset{u \in[0, \tau]}{\operatorname{Max}} H(u)
$$

presque partout sur $\left[0, t_{f}\right]$. $n^{\circ}$ V-1, 1972. 
Les relations (23)-(28) constituent le principe du maximum le long d'une réponse optimale à partir de l'état initial $\left(x^{\circ}, 0\right)$ et étant donnée la cible $\Xi_{2}^{*}$. D'après la proposition établie au paragraphe précédent cette cible elle-même est définie au voisinage de $\tilde{x}^{*}\left(t_{f}\right)$ par la relation :

$$
\frac{\partial \Phi_{2}}{\partial t}-\frac{\partial \Phi_{2}}{\partial x} s_{1}^{*}(x)\left(x-d^{*}\right)=0
$$

satisfaite en tout point de $\Xi_{2}^{*}$ dans ce voisinage. Nous avons posé le problème dans les termes classiques de la théorie de la commande optimale et des jeux différentiels. La résolution des équations (17) et (24) soumises aux conditions (23), (25)-(29) nous permet de caractériser une réponse optimale de façon équivalente à celle proposée dans la première partie de cet article.

\section{BIBLIOGRAPHIE}

[1] O. Ashenfelter and G. E. Johnson, «Bargaining Theory, Trade Unions, and Industrial Strike Activity », American Economic Review, 1969, pp. 35, 49.

[2] R. L. Bishop, «A Zeuthen-Hicks Theory of Bargaining », Econometrica, 1964, pp. 410-417.

[3] A. Blaquiere et G. Leitmann, Jeux quantitatifs, Memorial des Sciences Mathématiques. Gauthier-Villars, Paris, 1969.

[4] A. Blaquiere, "Quelques aspects géométriques des processus optimaux ", Cours tenu à Bressanone en juin 1966. Éditions Cremonèse, Rome.

[5] A. E. BRYson et Y. C. Ho, Applied Optimal Control, G. Blaisdell, 1969.

[6] J. CASE, "Towards a Theory of Many Player Differential Games ", Siam J. Control, vol. 7, no 2, 1969.

[7] J. H. CASE, "A Bargaining Model for Industrial Wage Disputes », Econometric Research Program-Research Memorandum, $\mathrm{n}^{\circ}$ 113, Princeton University, juin 1970.

[8] B. Contin,, «The Value of Time in Bargaining Negotiations : some experimental Evidence ", American Economic Review, 1968, pp. 374-393.

[9] B. Contrn, "The Value of Time in Bargaining Negotiations : A dynamic Model », Kyklos, 1970, pp. 255-271.

[10] J. G. Cross, "A Theory of the Bargaining Process », American Economic Review, 1965, pp. 67-94.

[11] A. Haurie, "Jeux quantitatifs à $M$ joueuirs ", Thèse ( $3^{e}$ cycle), Paris, 1970.

[12] R. IsAaCs, Differential Games, J. Wiley, New-York, 1965.

[13] G. LeitmanN, An Introduction to Optimal Control, McGraw-Hill, 1966.

Revue Française d'Automatique, Informatique et Recherche Opérationnelle no V-1, 1972. 